Ann. Zootech., I962, 11 (3), 217-224

\title{
L'APPRÉCIATION DE LA QUALITÉ DES CARCASSES BOVINES PAR LA DÉCOUPE DU MORCEAU TRICOSTAL 7, 8, 9,
}

\author{
J. MARTIN et G. TORREELE \\ Centre d'Études bovines, Coupure 235, Gand (Belgique) (1)
}

SOMMAIRE

Sur les bases de la découpe de demi-carcasses de jeunes taureaux à l'engrais, les relations entre la composition en viande, graisse et os de la moitié droite et la composition en viande, graisse et os du morceau tricostal 7,8 , 9 ont été établies.

Pour l'ensemble des $\mathbf{1} 7$ animaux examinés, ces relations sont estimées par :

$$
\begin{aligned}
& \text { p. } \left.100 \mathrm{M}_{\mathrm{H}^{0}}=15,9+0,82 \text { (p. 100 } \mathrm{M}_{\mathrm{R}^{\circ}}\right) \quad r^{2}=0,95 \\
& \text { p. Iоо } \mathrm{F}_{\mathrm{H}^{\circ}}=0,7+0,84\left(\text { p. гоо } \mathrm{F}_{\mathrm{R}^{0}}\right) \quad r^{2}=0,949 \\
& \text { p. } 100 \mathrm{~B}_{\mathrm{H}^{\circ}}=4,7+0,6\left(\text { p. } 100 \mathrm{~B}_{\mathrm{R}^{0}}\right) \quad r^{2}=0,67
\end{aligned}
$$

Dans ces équations, les variables dépendantes p. $100 \mathrm{M}_{\mathrm{H}^{\prime \prime}}$, p. $100 \mathrm{~F}_{\mathrm{H}^{\circ}}$ et p. $100 \mathrm{~B}_{\mathrm{I}^{\circ}}$ sont respectivement le pourcentage en viande, graisse et os dans la moitié droite de la carcasse ; les variables indépendantes p. $100 \mathbf{M}_{\mathbf{R}^{\circ}}$, p. Iо० $\mathrm{F}_{\mathrm{R}^{*}}$, p. $100 \mathrm{~B}_{\mathrm{R}^{\circ}}$ le pourcentage en viande graisse et os dans le morceau tricostal $7,8,9$.

A partir de ces équations de régression et des résultats de la découpe de go morceaux tricostaux, l'estimation de la variabilité dans la composition des carcasses de jeunes taureaux à l'engrais, appartenant à une population de bétail à deux fins, a donné : pourcentage en viande dans la carcasse : de 57 à $73,6 \mathrm{p}$. Ioo; pourcentage en graisse dans la carcasse : de Ir, 5 à $28,7 \mathrm{p}$. roo; pourcentage en os dans la carcasse : de ${ }_{13}, \mathrm{I}$ à i $7,7 \mathrm{p}$.

\section{INTRODUCTION}

L'élément les plus important dans la détermination de la valeur de boucherie d'un animal est sans aucun doute la composition quantitative de la carcasse. Quels que soient les buts et les méthodes d'une découpe de la carcasse, il est toujours possible de ramener cette découpe aux trois éléments constitutifs notamment la viande, les os et la graisse.

(1) Groupe de travail du Ministìre de l'Agriculture - Administration de la Recherche Agronomique. 
Parmi ces trois éléments, seule la musculature contribue dans un sens positif à la valeur marchande de la carcasse. Elle le fait en premier lieu par sa quantité, en second lieu par sa qualité. Nous nous bornons dans cette étude à l'aspect quantitatif du problème.

En considérant, d'une part, l'insuffisance des estimations par mensuration (DUMONT et IE CUEITE, I96r ; HERTRAMPF, I96r) et, d'autre part, en nous inspirant de différents travaux déjà entrepris dans ce domaine (Hankins et Hown, r946; Crown et Damon, ig6o ; Col,e, Orme et Kincaid, ig60; Kruger et Meyer, i960; WENIGER, I959) nous nous sommes adressés au morceau tricostal 7,8, 9 de la moitié droite pour en étudier la valeur comme échantillon représentatif de la carcasse. Visà-vis de la découpe du morceau tricostal, 9, IO, II, le prélèvement du morceau tricostal $7,8,9$ présente à notre avis l'avantage d'être mieux reproductible. Enn effet, il nous semble plus facile de faire une coupe reproductible entre la $9^{\mathbf{e}}$ et la IO $^{\mathrm{e}}$ côte qu'entre la $\mathrm{II}^{\mathrm{e}}$ et la $\mathrm{I}^{\mathrm{e}}$ du fait que la IO $^{\mathrm{e}}$ côte aboutit plus près du sternum que la $12^{\mathrm{e}}$. L'intersection entre $1 \mathrm{a} 9^{\mathrm{e}}$ et $1 \mathrm{la} \mathrm{Io}^{\mathrm{e}}$ côte constitue de ce fait un meilleur guide pour le couteau lors de la section du morceau tricostal.

\section{MATÉRIEL ET MÉTHODES}

L'ensemble des animaux examinés est constitué comme suit :

II taureaux engraissés intensivement et abattus à un poids final d'environ $495 \mathrm{~kg}$,

4 taureaux engraissés intensivement et abattus à un poids final voisin de $45^{\circ} \mathrm{kg}$,

I taureau cul-de-poulain de $48 \mathrm{r} \mathrm{kg}$, acheté dans le commerce,

I génisse de bonne conformation pesant $495 \mathrm{~kg}$, achetée dans le commerce.

Le poids final est le poids à la fin de l'engraissement ; le poids avant l'abattage est le poids tel qu'il est constaté sur la bascule officielle de l'abattoir de Gand, immédiatement avant l'abattage. Les pesées et les découpes sur la moitié droite ont lieu après un refroidissement d'environ i 8 heures

La séparation quartier avant-quartier arrière s'effectue par une section passant entre la $6^{\mathbf{e}}$ et la $7^{\mathrm{e}}$ côte. Une section passant entre la $9^{\mathrm{e}}$ et la $10^{\mathrm{e}}$ côte détache du quartier postérieur le morceau tricostal $7,8,9$.

La moitié droite ainsi que le morceau tricostal 7,8 , 9 sont divisés en viande, os et graisse + tendons. Pour ce faire, les tendons et toute la graisse superficielle et intermusculaire visible sont enlevés, de même la viande est soigneusement séparée des os.

Comme variables indépendantes figurent les pourcentages en viande (p. $100 \mathrm{M}_{\mathrm{R}^{\circ}}$ ) ; graisse (p. $100 \mathrm{~F}_{\mathrm{R}^{\circ}}$ ) et os $\left(\mathrm{p} .100 \mathrm{~B}_{\mathrm{R}^{\circ}}\right.$ ) dans le murceau tricostal 7, 8, 9. Les variables dépendantes sont les pourcentages en viande $\left(\mathrm{p}\right.$. 1 $100 \mathrm{M}_{\mathrm{H}^{\circ}}$ ) ; graisse (p. $100 \mathrm{~F}_{\mathrm{H}^{\circ}}$ ) et os $\left(\mathrm{p}, 100 \mathrm{~B}_{\mathrm{H}^{\circ}}\right)$ dans la moitié droite de la carcasse.

\section{RÉSUL'TATS E'T DISCUSSIONS}

Dans le tableau I se trouvent les chiffres absolus et relatifs obtenus lors de l'abattage de I7 animaux et de la découpe de la moitié droite des carcasses.

Le tableau 2 représente les chiffres absolus et relatifs obtenus lors de la découpe du morceau tricostal 7,8,9 de la moitié droite des carcasses. 
TABLEAU I

Observations concernant l'abattage et la découpe de la moitié droite des carcasses (I 7 animaux)

\begin{tabular}{|c|c|c|c|c|c|c|c|c|c|c|c|}
\hline & $\mathrm{W}_{s}$ & $\mathrm{R}_{s}$ & $\mathrm{H}^{\circ}$ & Mo & Fo & $\mathrm{B}^{\circ}$ & $\% M^{0}$ & $\% F^{o}$ & $\% B^{\circ}$ & $\mathrm{R}_{\mathbf{M}}$ & $\mathrm{M}$ \\
\hline & 476,0 & 61,8 & 146,5 & $9 \div, 2$ & 29,0 & 23,3 & 64,3 & 19,8 & 15,9 & 39,7 & 189 \\
\hline & 468,0 & 61,5 & 143,5 & 96,1 & 24,4 & 23,0 & 67,0 & 17,0 & 16,0 & 41,2 & 193 \\
\hline & 472,0 & 61,3 & 145,0 & 99,4 & 21,0 & 24,6 & 68,5 & 14,5 & 17,0 & 42,0 & 198 \\
\hline & 482,0 & 63,2 & 151,5 & 95,4 & 33,3 & 22,8 & 63,0 & 22,0 & 15,0 & 39,8 & 192 \\
\hline & 473,0 & 60,9 & 143,0 & 95,5 & 24,8 & 22,8 & 66,8 & 17,3 & 15,9 & 40,7 & 192 \\
\hline & 487,0 & 64,5 & 156,0 & 105,6 & 27,7 & 22,7 & 67,7 & 17,8 & 14,5 & 43,7 & 213 \\
\hline & 489,0 & 62,7 & 152,0 & 96,7 & 32,0 & 23,3 & 63,6 & 21,1 & 15,3 & 39,9 & 195 \\
\hline & 495,5 & 62,1 & 152,0 & $94, l_{4}$ & 33,2 & 24,4 & 62,1 & 21,8 & 16,1 & 38,5 & 191 \\
\hline & $47 \%, 0$ & 61,7 & 145,5 & 83,1 & 41,1 & 21,3 & 57,1 & 28,3 & 14,6 & 35,2 & 167 \\
\hline & 487,5 & 62,5 & 152,0 & 90,0 & 40,8 & 21,2 & 59,2 & 26,8 & 14,0 & 37,0 & 180 \\
\hline & 477,0 & 63,3 & 151,0 & 107,6 & 21,3 & 22,1 & 71,3 & 14,1 & 14,6 & $45, \mathrm{t}$ & 215 \\
\hline \multirow[t]{5}{*}{$a$} & 480,0 & 62,32 & 148,91 & 96,18 & 29,87 & 22,86 & 64,6 & 20,04 & 15,36 & 40,25 & 193,2 \\
\hline & 453,3 & 63,1 & 142,0 & 93,1 & 27,2 & 21,7 & 65,6 & 19,2 & 15,2 & $/ 1,4$ & 187 \\
\hline & $4\{3,5$ & 61,9 & 137,0 & 87,7 & 28,5 & 20,8 & 64,0 & 20,8 & 15,2 & 39,6 & 176 \\
\hline & 447,5 & 61,2 & 136,5 & 89,3 & 27,9 & 19,3 & 65,4 & 20,5 & $1:, 1$ & 40,1 & 179 \\
\hline & 436,0 & 60,6 & 132,0 & 85,7 & 24,2 & 22,1 & 64,9 & 18,3 & 16,8 & 39,3 & 171 \\
\hline$b$ & $4 / 5,0$ & 61,7 & 136,87 & 88,95 & 26,95 & 20,97 & 64,97 & 19,67 & 15,3 & 40,1 & 178,2 \\
\hline$c$ & 470,73 & 62,16 & 145,7 & 94,26 & 29,09 & 22,36 & 64,7 & 19,94 & 15,34 & 40,21 & 189,2 \\
\hline 0 & 481,0 & 66,0 & 159,0 & 126,2 & 12,9 & 20,0 & 79,3 & 8,1 & 12,6 & 52,4 & 252 \\
\hline 00 & 495,0 & 60,8 & 149,0 & 97,6 & 30,3 & 21,2 & 65,5 & 20,3 & 14,2 & 39,8 & 197 \\
\hline
\end{tabular}

$\mathrm{W}_{s}$ : Poids avant l'abattage.

$\mathrm{R}_{s}$ : Rendement à l'abattage.

$\mathrm{H}^{\circ}$ : Poids de la moitié droite de la carcasse.

Mo : $\mathrm{kg}$ de viande dans la moitié droite de la carcasse.

$F^{0}:$ : $\mathrm{kg}$ de graisse dans la moitié droite de la carcasse.

$\mathrm{B}^{0}$ : $\mathrm{kg}$ d'os dans la moitié droite de la carcasse.

$\% \mathrm{M}^{\circ}, \% \mathrm{Fo}^{\circ}, \% \mathrm{~B}^{\mathrm{o}}$ resp. pourcentage en viande, graisse et os dans la moitié droite.

$\mathrm{R}_{\mathrm{M}}$ : Coefficient de production en viande $=\mathrm{R}_{s} \times \% \mathrm{M}$.

M : Production totale en viande $(\mathrm{kg})$.

$a$ : Les moyennes pour les 11 taureaux de $\pm 495 \mathrm{~kg}$.

$b$ : Les moyennes pour les 4 taureaux de $\pm 450 \mathrm{~kg}$.

$c$ : Les :noyennes pour les 15 taureaux engraissés intensivement.

o : cul-de-poulain.

oo : Génisse. 
TABIEAU 2

Résultats de la découpe du morceau tricostal ( 17 animaux)

\begin{tabular}{|c|c|c|c|c|c|c|c|c|}
\hline & $\mathrm{R}^{0}$ & $\frac{\mathrm{R}^{\circ}}{\mathrm{H}^{0}} \times 100$ & $\mathrm{M}_{\mathrm{R}^{0}}$ & $F_{R^{\circ}}$ & $\mathrm{B}_{\mathrm{R}^{\circ}}$ & $\% \mathbf{M}_{\mathbf{R}^{0}}$ & $\% \mathrm{~F}_{\mathrm{R}^{\circ}}$ & $\% \mathrm{~B}_{\mathrm{n}^{\circ}}$ \\
\hline & 9,95 & 6,79 & 5,70 & 2,46 & 1,79 & 57,29 & 24,72 & 17,99 \\
\hline & 9,63 & 6,71 & 5,91 & 1,83 & 1,89 & 61,36 & 19,01 & 19,63 \\
\hline & 9,60 & 6,62 & 6,13 & 1,65 & 1,82 & 63,85 & 17,19 & 18,96 \\
\hline & 10,70 & 7,06 & 6,31 & 2,57 & 1,82 & 58,97 & 24,02 & 17,01 \\
\hline & 9,10 & 6,36 & 5,52 & 1,95 & 1,63 & 60,66 & 21,43 & 17,91 \\
\hline & 10,45 & 6,70 & 6,40 & 2,25 & 1,80 & 61,24 & 21,54 & 17,22 \\
\hline & 10,90 & 7,17 & 6,27 & 2,63 & 2,00 & 57,52 & 24,13 & 18,35 \\
\hline & 10,20 & 6,71 & 5,75 & 2,43 & 2,02 & 56,37 & 23,82 & 19,81 \\
\hline & 10,70 & 7,35 & 5,43 & 3,53 & $1,7 t$ & 50,75 & 32,99 & 16,26 \\
\hline & 11,30 & 7,43 & 6,15 & 3,35 & 1,80 & $5 / 4,42$ & 29,65 & 15,93 \\
\hline & 10,70 & 7,09 & 7,16 & 1,71 & 1,83 & 66,92 & 15,98 & 17,10 \\
\hline \multirow[t]{5}{*}{$a$} & 10,293 & 6,908 & 6,066 & 2,396 & 1,830 & 59,036 & 23,13 & 17,832 \\
\hline & 10,10 & 7,11 & 6,27 & 2,16 & 1,67 & 62,08 & 21,39 & 16,53 \\
\hline & 8,80 & 6,42 & 5,08 & 2,12 & 1,60 & 57,73 & 24,09 & 18,18 \\
\hline & 10,10 & 7,40 & 6,10 & 2,20 & 1,80 & 60,40 & 21,78 & 17,82 \\
\hline & 8,70 & 6,59 & 5,10 & 2,02 & 1,58 & 58,62 & 23,22 & 18,16 \\
\hline$b$ & 9,425 & 6,88 & 5,638 & 2,125 & 1,663 & 59,708 & 22,62 & 17,672 \\
\hline$c$ & 10,062 & 6,90 & 5,952 & 2,324 & 1,786 & 59,215 & 22,994 & 17,79 \\
\hline 0 & 11,15 & 7,01 & 8,67 & 0,91 & 1,57 & 77,76 & 8,16 & 14,08 \\
\hline oo & 11,65 & 7,82 & 7,30 & 2,60 & 1,75 & 62,66 & 2232 & 15,02 \\
\hline
\end{tabular}

Ro : Poids du morceau tricostal 7,8,9(kg) ;

$\mathrm{R} / \mathrm{II}^{\circ} \times 100:$ Pourcentage du morceau tricostal vis-à-vis de la moitié droite ;

$\mathrm{M}_{\mathrm{R}^{\circ}} \quad$ : Kg de viande dans le morceau tricostal ;

$\mathrm{F}_{\mathrm{R}^{\circ}} \quad$ : Kg de graisse dans le morceau tricostal ;

$\mathrm{B}_{\mathrm{R}^{\mathrm{n}}} \quad: \mathrm{Kg}$ d'os dans le morceau tricostal ;

$\% \mathrm{H}_{\mathrm{R}^{\circ}}, \% \mathrm{~F}_{\mathrm{R}^{\mathrm{N}}}, \% \mathbf{B}_{\mathrm{R}^{\circ}}$ : resp. pourcentage en viande, graisse et os dans le morceau tricostal ;

a : les moyennes pour les 11 taureaux de $\pm 495 \mathrm{~kg}$;

$b \quad:$ les moyennes pour les 4 taureaux de $\pm 450 \mathrm{~kg}$;

$c \quad:$ les moyennes pour les 15 taureaux engraissés intensivement;

o cul-de-poulain ;

oo : génisse.

Dans le tableau 3 sont repris les pourcentages en viande, graisse et os obtenus d'une part sur le morceau tricostal $7,8,9$; d'autre part sur la moitié droite de la même carcasse. En même temps y figurent les estimations obtenues à partir d'une régression linéaire basée sur les observations faites sur les II taureaux d'environ $45^{\circ} \mathrm{kg}$.

Les données du tableau 3 sont reprises dans le graphique $I$; en ordonnée les rendements en viande de la moitié droite ( $\mathrm{p}$. Ioo $\mathrm{M}_{\mathrm{H}^{\circ}}$ ) et en abscisse les rendements en viande du morceau tricostal des mêmes carcasses.

Calculée sur la base des données des II taureaux de $495 \mathrm{~kg}$, le coefficient de corrélation est de 0,977 . Les droites de régression calculées pour ces II animaux sont les suivantes:

$$
\begin{aligned}
& \text { p. } 100 \mathrm{M}_{\mathrm{H}^{\circ}}=10,62+0,9 \mathrm{I} 4 \text { (p. I00 } \mathrm{M}_{\mathrm{R}^{0}} \text { ) } \\
& \text { p. I00 } \left.\mathrm{F}_{\mathrm{H}^{0}}=0,53+0,889 \text { (p. IOO } \mathrm{F}_{\mathrm{R}^{\circ}}\right) \quad r=0,972 \\
& \text { p. Iо0 } \mathrm{B}_{\mathrm{H}^{\circ}}=4,7^{8}+0,593\left(\text { p. I00 } \mathrm{B}_{\mathrm{K}^{n}}\right) \quad r=0,843
\end{aligned}
$$


TABLEAU 3

Composition relative du morceau tricostal et de la moitié droite

\begin{tabular}{|c|c|c|c|c|c|c|c|c|}
\hline \multicolumn{3}{|c|}{$\%$ de viande } & \multicolumn{3}{|c|}{$\%$ de graisse } & \multicolumn{3}{|c|}{$\%$ d'os } \\
\hline $\mathrm{R}^{\circ}$ & $\mathrm{H}^{\circ}$ & Est. & $\mathrm{R}^{0}$ & $\mathrm{H}^{\circ}$ & Est. & $\mathrm{R}^{0}$ & $\mathrm{H}^{\mathrm{o}}$ & Est. \\
\hline 57,3 & 64,3 & 63,0 & 24,7 & 19,8 & 21,4 & 18,0 & 15,9 & 15,5 \\
\hline 61,4 & 67,0 & 66,7 & 19,0 & 17,0 & 16,4 & 19,6 & 16,0 & 16,4 \\
\hline 63,9 & 68,5 & 69,0 & 17,2 & 14,5 & 14,8 & 19,0 & 17,0 & 16,0 \\
\hline 59,0 & 63,0 & 64,5 & 24,0 & 22,0 & 20,8 & 17,0 & 15,0 & 14,9 \\
\hline 60,7 & 66,8 & 66,1 & 21,4 & 17,3 & 18,5 & 17,9 & 15,9 & 15,4 \\
\hline 61,2 & 67,7 & 66,6 & 21,5 & 17,8 & 18,6 & 17,2 & 14,5 & 15,0 \\
\hline 57,5 & 63,6 & 63,2 & 24,1 & 21,1 & 20,9 & 18,4 & 15,3 & 15,7 \\
\hline 56,4 & 62,1 & 62,2 & 23,8 & 21,8 & 20,6 & 19,8 & 16,1 & 16,5 \\
\hline 50,8 & 57,1 & 57,0 & 33,0 & 28,3 & 28,8 & 16,3 & 14,6 & 14,4 \\
\hline 54,4 & 59,2 & 60,4 & 29,7 & 26,8 & 25,9 & 15,9 & 14,0 & 14,3 \\
\hline 66,9 & 71,3 & 71,8 & 16,0 & 14,1 & 13,7 & 17,1 & 14,6 & 14,9 \\
\hline 62,1 & 65,6 & 67,4 & 21,4 & 19,2 & 18,5 & 16,5 & 15,2 & 14,6 \\
\hline 57,7 & 64,0 & $63,{ }^{\prime}$ & 24,1 & 20,8 & 20,9 & 18,2 & 15,2 & 15,6 \\
\hline 60,4 & 65,4 & 65,8 & 21,8 & 20,4 & 18,9 & 18,8 & 14,1 & 16,0 \\
\hline 58,6 & 64,9 & 64,2 & 23,2 & 18,3 & 20,1 & 18,2 & 16,7 & 15,6 \\
\hline 77,8 & 79,4 & 81,7 & 8,2 & 8,1 & 6,8 & 14,1 & 12,6 & 13,2 \\
\hline 62,7 & 65,5 & 67,9 & 22,3 & 20,3 & 19,3 & 15,0 & 14,2 & 13,7 \\
\hline
\end{tabular}

$\mathrm{R}^{\circ}$ : morceau tricostal 7, 8, 9 de la moitié droite de la carcasse ;

$\mathrm{H}^{\mathrm{o}}$ : moitié droite de la carcasse ;

Est. : estimation d'après la dissection du morceau tricostal.

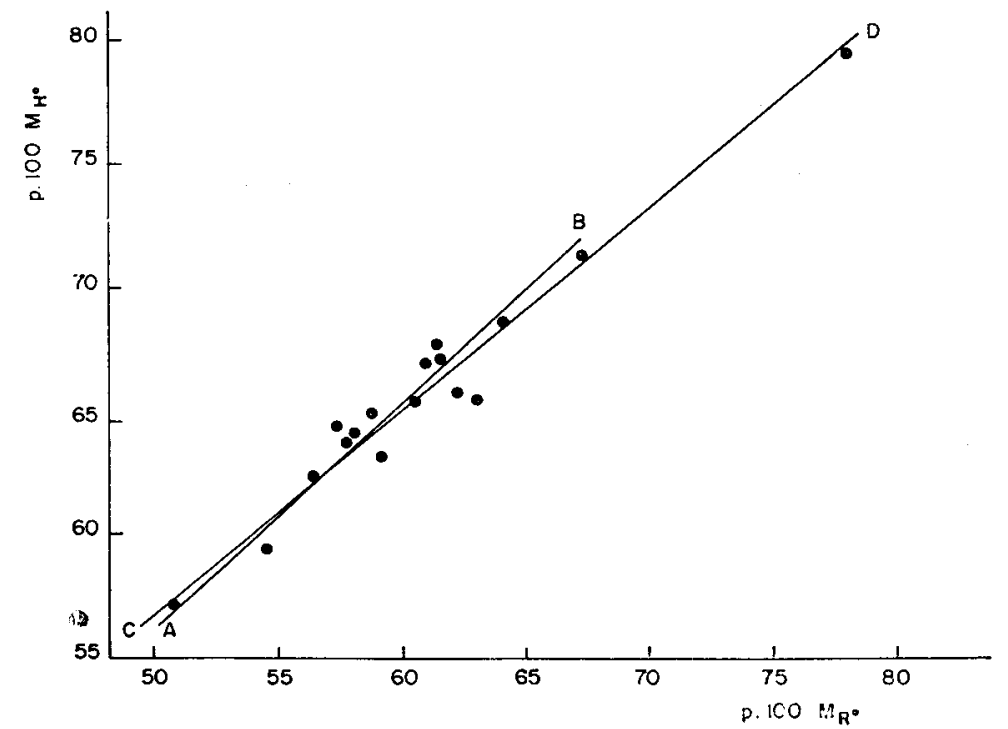

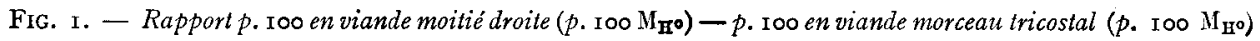

p. $100 \mathrm{M}_{\mathrm{H}^{\circ}}=15,90+0,82$ p. I00 $\mathrm{M}_{\mathrm{R}}$ (droite $\mathrm{CD}, \mathrm{I} 7$ animaux)

p. $100 \mathrm{M}_{\mathrm{H}^{\circ}}=10,62+0,914$ p. $100 \mathrm{M}_{\mathrm{R}^{\circ}}$ (droite $\mathrm{AB}$, i animaux) 
Il s'avère que la droite de régression $A B$ garde sa valeur pour les 4 taureaux de $450 \mathrm{~kg}$, pour le cul-de-poulain de $48 \mathrm{I} \mathrm{kg}$ et pour la génisse de $495 \mathrm{~kg}$. Notons toutefois qu'il s'agit dans tout les cas d'animaux en conditions d'abattage.

Pour l'ensemble des $\mathrm{I} 7$ animaux examinés, la droite de régression estimant les pourcentages en viande de la moitié droite par ceux observés sur le morceau tricostal est (droite CD du graphique I)
p. Io0 $\mathrm{M}_{\mathrm{H}^{\circ}}=\mathrm{I} 5,9+0,82$
(p. roo $\mathrm{M}_{\mathrm{R}^{\circ}}$ )
$n=\mathrm{I} 7 ; r^{2}=0,956$

A titre d'exemple, nous donnons ci-dessous les couples (pourcentage en viande observé dans la moitié droite; valeur calculée) pour les I7 animaux examinés.

$\begin{array}{lllll}(64,3 ; 62,9), & (67,0 ; 66,2), & (68,5 ; 68,3), & (63,0 ; 64,3), & (66,8 ; 65,6), \\ (67,7 ; 66, \mathrm{I}), & (63,6 ; 63, \mathrm{I}), & (62, \mathrm{I} ; 62, \mathrm{I}), & (57,1 ; 57,5), & (59,2 ; 60,5), \\ (71,3 ; 70,8), & (65,6 ; 66,8), & (64,0 ; 63.8), & (65,4 ; 65,4), & (64,9 ; 64,0), \\ (79,4 ; 79,7), & (65,5 ; 67,3), & & & \end{array}$

Les droites de régression estimant les pourcentages en graisse et en os de la moitié droite par ceux observés sur le morceau tricostal sont respectivement:
p. I00 $\mathrm{F}_{\mathrm{H}^{\circ}}=0,7+0,84\left(\right.$ p. I00 $\left.\mathrm{F}_{\mathrm{R}^{\circ}}\right)$
$n=\mathrm{I} 7 ; r^{2}=0,95$
p. I00 $\mathrm{B}_{\mathrm{H}^{\circ}}=4,7+0,60\left(\mathrm{p}\right.$. I00 $\left.\mathrm{B}_{\mathrm{R}^{\circ}}\right)$
$n=\mathrm{I} 7 ; r^{2}=0,67$

Un autre problème également très important a été étudié à la même occasion, l'étendue de la variation du pourcentage des trois éléments constitutifs des carcasses.

Les II taureaux de $495 \mathrm{~kg}$, quoique appartenant à la même population et engraissés dans des conditions sensiblement égales (même ration, même étable, même soins) présentaient de leur vivant une qualité de boucherie très différente ce qui d'ailleurs s'est confirmée à la découpe des carcasses. A consulter le tableau 2 on constate que le rapport en viande des carcasses varie de $57, \mathrm{I}$ p. Ioo à $7 \mathrm{I}, 3$ p. Ioo, le rapport en graisse varie de I4, I p. Ioo à $28,3 \mathrm{p}$. Ioo, tandis que les pourcentages en os oscillent entre $I 4,5$ p. Ioo et I7,0 p. IOO, c'est à-dire se maintiennent aux environs de I5,5 p. IOO.

I a somme des trois pourcentages étant égale à roo p. Ioo, il est possible de représenter les résultats dans un diagramme triangulaire (voir graphique 2 ). Chaque point alors représente une carcasse et la situation de chaque point nous renseigne au sujet du rapport en viande, graisse et os des carcasses.

On constate en même temps que le rapport en viande d'une carcasse de culde-poulain est nettement supérieur au meilleur animal de conformation normale.

L,es équations de régression calculées sur la base des données des in taureaux de $495 \mathrm{~kg}$ permettent de calculer et d'étudier de plus près la variation provenant de 90 taureaux engraissés dans des conditions comparables jusqu'a un poids constant de $\pm 495 \mathrm{~kg}$. Ces taureaux, appartenant principalement à une même population de bétail à 2 fins, ont été mis à l'engraissement à partir de l'âge de \pm I 5 jours et ont été nourris à un régime intensif comparable comprenant essentiellement une alimentation ad libitum de concentré et de foin.

Les données ainsi obtenues sont représentés dans le graphique 3 .

Ces données permettent de constater que dans une population de bétail à deux fins, il se présente dans les éléments constitutifs de la carcasse, une variation assez large à tel point qu'une sélection des qualités de boucherie semble prometteuse. 


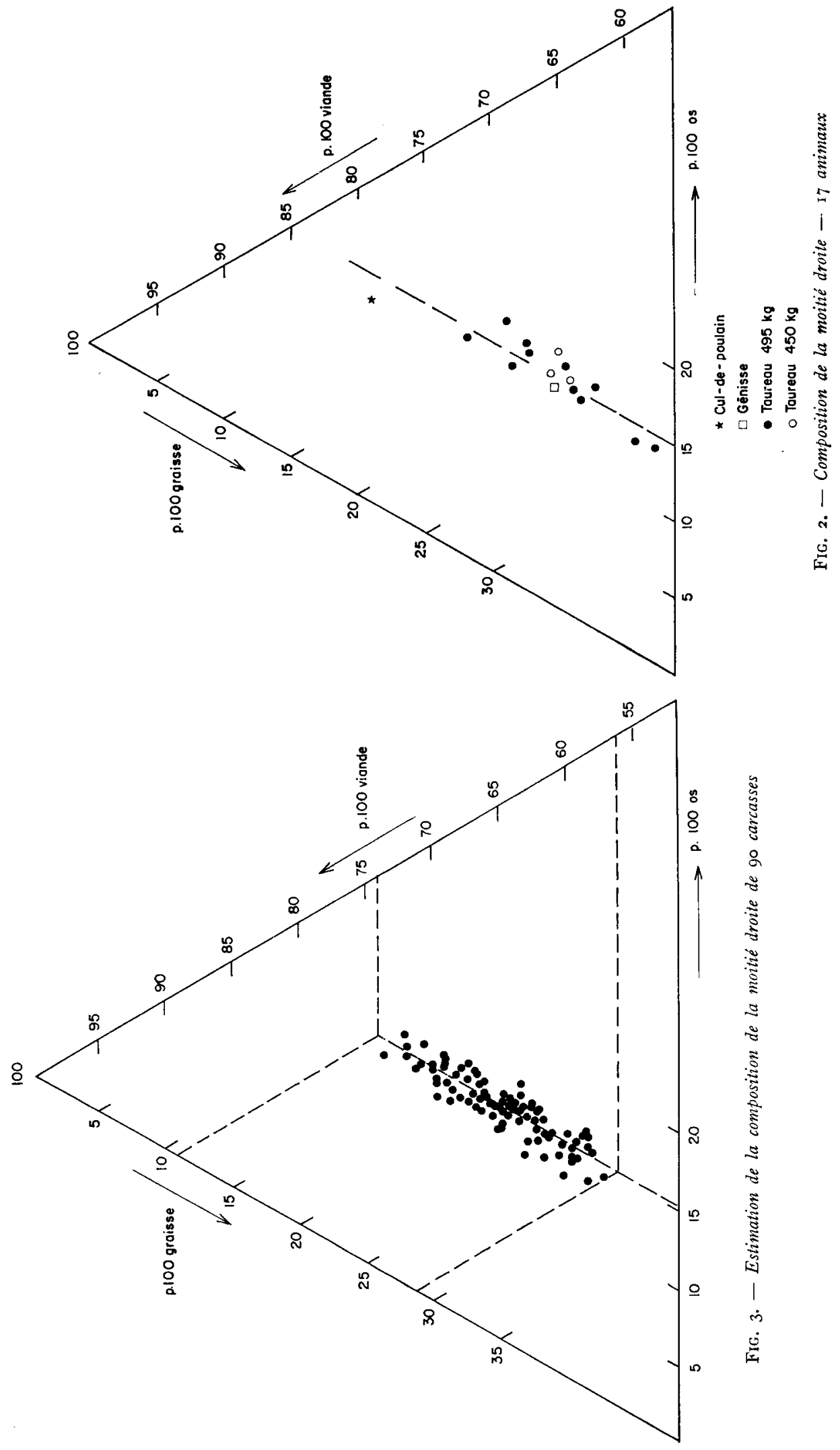


La variation ainsi constatée peut se résumer comme suit:

$\begin{array}{lccc} & & \text { morceau tricostal } & \text { carcasse } \\ \% \text { en viande } \ldots \ldots \ldots \ldots \ldots & \mathbf{5 0 , 8}-68,9 & \mathbf{5 7 , 0 5}-\mathbf{7 3 , 5 9} \\ \% \text { en graisse } \ldots \ldots \ldots \ldots \ldots & 13,5-32,9 & \mathbf{1 1 , 4 8}-28,73 \\ \% \text { en os } \ldots \ldots \ldots \ldots \ldots \ldots & 14,0-21,7 & \mathbf{1 3 , 1 1}-\mathbf{1 7 , 6 5}\end{array}$

Ręu pour publication en octobre 1962.

\section{SUMMARY}

AN ESTIMATION OF CARCASS QUALITY BY TIIE DISSECTION OF THE THREE RIH CUT 7. 8.9.

The separable lean, fat and bone of the three rib cut $7,8,9$ were evaluated as to their value for predicting separable lean, fat and bone content of the entire carcass. For the set of 17 animals investigated, the following regression equations were obtained :

p. $100 \mathrm{M}_{\mathrm{H}^{\circ}}=15.9+0.82\left(\right.$ p. $\left.100 \mathrm{M}_{\mathrm{R}^{\prime \prime}}\right) ; \quad$ p. $100 \mathrm{~F}_{\mathrm{H}^{0}}=0.07+0.84\left(\right.$ p. $\left.100 \mathrm{~F}_{\mathrm{H}^{0}}\right)$;

p. $100 \mathrm{~B}_{\mathbf{H}^{\circ}}=4.7+0.60$ (p. $\left.100 \mathrm{~B}_{\mathbf{R}^{\circ}}\right)$.

In this equations, p. $100 \mathrm{M}_{\mathrm{H}^{\circ}}$, p. $100 \mathrm{~F}_{\mathrm{H}^{\circ}}$ and p. $100 \mathrm{~B}_{\mathrm{Ir}^{\circ}}$ stand for the percentages of lean, fat and bone in the carcass halve while p. $100 \mathrm{M}_{\mathrm{R}^{0}}, \mathrm{p}$. 100 $\mathrm{F}_{\mathrm{R}^{0}}$ and $\mathrm{p}$. $100 \mathrm{~B}_{\mathrm{R}^{\circ}}$ stand for the percentages of lean, fat and bone in the three rib cut $7,8,9$. The coefficients of determination were respectively $0.95,0.95$ and 0.67 .

From this relations and the dissection results of go three rib cuts, an estimation of the variability in carcass composition within a two purpose breed gave the following ranges of variation : percentage of lean in the carcass $57.0-73.6 \mathrm{p}$. 100; percentage of fat in the carcass : $11.5-28.7$ p. I00; percentage of bone in the carcass : I3.1 -17.7 p. 100.

\section{RÉFÉRENCES BIBLIOGRAPHIQUES}

Dumont B. L., Le Guelte P., r961. La Conformation du Bovin de Boucherie. Rapp. Ass. prof. Fabricants d'Aliments composés pour Animaux, Bruxelles.

Hankins O. G., Howe P. E., 1946. Estimation of the composition o ibeef carcasses and cuts. Tech. Bull. U.S. Dep. Agric., n ${ }^{\circ} 926$.

Crows R. M., Dinon R. A., 1960. The value of the isth rib out for measuring beef carcass yields and meat quality. J. Anim. Sci., 19, 109-113.

Cole J. W., Orme L. E., Kincaid C. M., I960. Relationship of loin eye area, separable lean of various beef cuts and carcass measurements to total carcass lean in beef. J. Anim. Sci., 19, 89-100.

KrUger I., Meyer F., i 960 . Untersuchungen an geschlachteten Jungmastbullen. Züchtungskunde, 32 .

HertrampF J., ig6r. Schlachtkörperbewertung beim Rind. Z. Tierz. Züchtbiol., 75, 276-292.

Weniger J. H., 1959. Probleme zur Erfassung von Schlachtwert und Schlachtqualität bein Rind. Arb. dtsch. Lander Ges., 63. 